\title{
Effects of endostatin production on oncogenicity and metastatic activity of HPV16-transformed mouse cells: Role of interleukin $1 \alpha$
}

\author{
MONIKA LAKATOSOVA-ANDELOVA, MARTINA DUSKOVA, VINCENT LUCANSKY, \\ PETR PARAL and VLADIMIR VONKA \\ Department of Experimental Virology, Institute of Haematology and Blood Transfusion, \\ U Nemocnice 1, Prague, Czech Republic
}

Received February 12, 2009; Accepted April 16, 2009

DOI: 10.3892/ijo_00000331

\begin{abstract}
Two mouse HPV16-transformed cell lines, viz. MK16 cells, which induce metastasizing tumors, and TC-1 cells, which induce non-metastasizing tumors were transduced with the gene for mouse endostatin. Two clones constitutively expressing endostatin were isolated from each of them. They were denoted ME3 and ME9, and TE2 and TE5, respectively. When inoculated into mice, ME3 cells were non-oncogenic. Nearly all mice inoculated with ME9 cells developed tumors, but considerably later than did the parental MK16 cells and metastasis formation was strongly reduced in these animals. On the other hand, TE2 and TE5 cells displayed oncogenic potential similar to that of the parental cells. To provide more information on these different effects of endostatin production, cell lysates of all six lines studied were tested for the content of 25 factors known to be involved in angiogenesis. The parental MK16 cells differed from the parental TC-1 cells and also from all endostatin producing sublines by a markedly higher production of interleukin $1 \alpha$ (IL-1 $\alpha$ ) and, to a lesser extent, by a higher production of several other factors tested. Additional experiments indicated that the suppression of the production of IL-1 $\alpha$ by the parental MK16 caused by endostatin was due to an autocrine mechanism.
\end{abstract}

\section{Introduction}

Tumor neoangiogenesis is a complex process including activation, migration and proliferation of endothelial cells, disruption of vascular basal membranes and formation of the new vascular networks. Its onset entails a disruption of the local equilibrium between pro-angiogenetic and anti-angiogenetic factors. In spite of great efforts, the process of angiogenesis

Correspondence to: Professor Vladimír Vonka, Department of Experimental Virology, Institute of Haematology and Blood Transfusion, U Nemocnice 1, Prague 2, Czech Republic E-mail:vonka@uhkt.cz

Key words: endostatin, HPV16-transformed cells, oncogenicity, metastasizing activity, IL- $1 \alpha$ has not yet been fully clarified and its somewhat detailed understanding on the molecular level has been achieved only recently. More than 50 factors acting either pro- or antiangiogenically have been identified and it is expected that some more will be discovered in the near future. Many of these substances are produced by tumor cells or other cell types present in the tumor environment. In clinical oncology, much attention is being paid to therapeutic approaches aiming at the prevention or reduction of neoangiogenesis. The attractiveness of this approach is substantiated by its wide spectrum of applicability, absence of drug resistance and low or nil toxicity. Furthermore, the complexity of the process of angiogenesis provides a number of targets for therapy. A growing number of anti-angiogenesis drugs are on clinical trials and many more are under development. Inter alia, gene-transfer methods are being employed to achieve these goals. All these efforts are not slowed down by a major weakness of anti-angiogenic therapy: It inhibits tumor growth but does not eliminate tumor cells.

Among the endogenous substances that have been found to inhibit neoangiogenesis and tumor growth, the one extensively studied is endostatin (ES), a $20 \mathrm{kDa}$ C-terminal, 184 amino acid-long proteolytic fragment of collagen XVIII (1). It is not yet known in detail how ES exerts its anti-angiogenetic activity. It apparently interacts with several receptors and initiates a complex network at the gene level that results in a downregulation of several signalling pathways associated with neoangiogenesis and an upregulation of those associated with the opposite effects. In human endothelial cells, it targets angiogenesis regulatory genes on $>12 \%$ of the human genome (2). Thus, ES acts as a specific inhibitor of endothelial cell proliferation and migration through multiple mechanisms such as the inhibition of metalloproteinases (3), the vascular endothelial cell-growth factor (VEGF) (4) or the fibroblastgrowth factor (FGF) (5) and the reduction of anti-apoptotic proteins, e.g. Bcl2 and $\mathrm{BclXL}$ (6). Of the endogenous inhibitors of angiogenesis it has the broadest anti-cancer spectrum, with virtually no toxicity (1).

It was the aim of the present undertaking to investigate the effect of ES production on the behaviour of tumor cells. As a model system, we chose two mouse (C57BL/6) oncogenic cell lines transformed by human papillomavirus type 16 (HPV16). Both are oncogenic for syngeneic animals, but they differ in a number of properties including the capability 
of metastizing (see below). Herein we show that ES production was associated with the loss or marked impairment of the oncogenic characteristics of the progenies of one, but not of the other, of the cell lines used and that these changes were associated with a decreased production of some angiogenic factors, primarily interleukin $1-\alpha$ (IL-1 $\alpha)$.

\section{Materials and methods}

Cell lines and media. The MK16 cell line was derived in our laboratory by the transformation of mouse C57BL/6 kidney cells via their co-transfection with the HPV16 E6 and E7 genes and the activated H-ras oncogene (7). TC-1 cells (obtained from T.C. Wu, Johns Hopkins University, Baltimore, MD, USA) have been isolated after transfection of mouse C57BL/6 lung cells with a similar set of genes (8). The two cell lines differ in a number of characteristics. TC-1 cells have fibroblastoid morphology, do not express cytokeratines and express MHC class I and B7.1 co-stimulatory molecules on their surface (9). They are highly oncogenic $\left(1 \mathrm{TID}_{50}\right.$ corresponds to $\sim 1 \times 10^{3}$ cells), but the subcutaneous tumors induced by them do not metastasize spontaneously. On the other hand, MK16 cells are of epitheloid morphology (8), they do form cytokeratins and are MHC class I and B7.1 negative. They are less oncogenic ( $1 \mathrm{TID}_{50}$ corresponds to $1 \times 10^{4}$ cells) than TC-1 cells, but the subcutaneous tumors induced by them do metastasize spontaneously to lymph nodes and lungs. It has also been shown that MK16 cells produce MHC class I molecules when cultivated in the presence of interferon- $\gamma$ IFN- $\gamma$ (7), as well as in vivo in the course of tumor growth (10). Both MK16 and TC-1 cells were cultivated in Dulbecco's modified Eagle's medium (D-MEM, BRL, Paisley, UK) containing $10 \%$ FCS (PAA Laboratiories, Linz, Austria), $100 \mathrm{U} / \mathrm{ml}$ penicillin, $100 \mu \mathrm{g} / \mathrm{ml}$ streptomycin and $2 \mathrm{mM} \mathrm{L}$ glutamine (Sevac, Prague, Czech Republic). Transfected cells were cultivated in medium supplemented with $15 \mu \mathrm{g} / \mathrm{ml}$ blasticidin (Cayla-InvivoGen Europe, France). Primary isolated human umbilical-vein endothelial cells (HUVEC, Cascade Biologics, UK) were cultivated in growth Medium 200 supplemented with LSGS kit (Cascade Biologics, UK). Medium 200 supplemented with LSGS kit will be referred to below as M200. Passages 3-7 were used for proliferation assays. All cells were incubated at $37^{\circ} \mathrm{C}$ in a $5 \% \mathrm{CO}_{2}$ atmosphere.

Transfection experiments. Plasmid pBLAST49-mEndo (CaylaInvivoGen Europe, France), which, in addition to blasticidine resistance gene, carries the murine ES gene linked to the interleukin-2 (IL-2) gene fragment encoding signal sequence for secretion, was propagated in the E. coli SURE strain in Fast-Media Blas TB medium (Cayla-InvivoGen Europe, France) and was purified with the use of the Qiagen plasmid maxi kit (Qiagen, Germany). Counts of $2.5 \times 10^{5}$ MK16 or TC-1 cells in $3 \mathrm{ml}$ complete D'MEM were seeded in 6-cm dishes (TPP AG, Trasadingen, Switzerland). One hour later, the cultures were transfected with $12 \mu \mathrm{g}$ plasmid DNA in HEPES buffered saline solution, using the modified calcium phosphate precipitation method. Following 24-h incubation the regular medium was replaced by $4 \mathrm{ml}$ of complete D'MEM supplemented with $15 \mu \mathrm{g} / \mathrm{ml}$ blasticidin. After 14-day cultivation the cells were harvested. Subsequently, cell clones were isolated and expanded in medium supplemented with blasticidin.

Counts of $2.5 \times 10^{5}$ cells in $3 \mathrm{ml}$ D'MEM with $10 \%$ FCS were seeded in $6-\mathrm{cm}$ dishes. The ES concentration in the medium was measured after 48-h incubation of the cells, using the ChemiKine ${ }^{\mathrm{TM}}$ Mouse Endostatin ${ }^{\mathrm{TM}}$ EIA kit (Chemicon $^{\mathrm{R}}$ International, Inc., USA). The amount of ES was expressed in $\mathrm{ng} / 10^{6}$ cells $/ 48 \mathrm{~h}$.

Flow cytometry. Counts of $0.5 \times 10^{6}$ trypsinized cells were washed twice with PBS. MHC class I molecules were detected after incubation of the cells with anti-mouse $\mathrm{H}-2 \mathrm{~K}^{\mathrm{b}} / \mathrm{H}-2 \mathrm{D}^{\mathrm{b}}$ monoclonal antibody (Pharmingen, San Diego, CA) or with isotype control antibody (Sigma, St. Louis, MO) at $4^{\circ} \mathrm{C}$ for 20-30 min. The cells were then washed in PBS and incubated with FITC-conjugated goat anti-mouse IgG antibody (Pharmingen). Fluorescence was measured with a flow cytometer (FACSCalibur, Beckman Dickinson). MK16 cells (MHC class I negative) and TC-1 cells (MHC class I positive) were used as negative and positive controls, respectively.

HUVEC proliferation assay. In this assay, media from cultures of the ME3, ME9, TE2 and TE5 cell lines and the parental MK16 and TC-1 cells, concentrated 20x with Centricon-10 concentrators (Millipore, Bedford, MA, USA), were used. Counts of $2.5 \times 10^{5}$ cells in $3 \mathrm{ml}$ of D-MEM medium with $10 \%$ FCS were seeded in 6-cm dishes and cultivated for $48 \mathrm{~h}$. The next day, $2 \times 10^{2}$ HUVEC cells in $100 \mu 1$ of M200 per well were seeded into 96-well plates (PAA Laboratories, Linz, Austria). After $24 \mathrm{~h}$, the M200 was replaced by $90 \mu \mathrm{l} /$ well of fresh M200 and $10 \mu \mathrm{l} /$ well of concentrated medium ( $2 \mathrm{ml}$ to $100 \mu \mathrm{l})$ removed from ME3, ME9, TE2, TE5, MK16 and TC-1 cultures. For each cell line four parallels were used. A similarly concentrated complete D'MEM medium $(10 \mu \mathrm{l})$ mixed with M200 medium $(90 \mu \mathrm{l})$ and added to HUVEC cells in four parallels served as a control. Concentrated D'MEM medium mixed with M200 medium (1+9) free of HUVEC cells was used for background subtraction. Every $24 \mathrm{~h}$, for four consecutive days, the media were replaced by fresh media of the same composition. Sensitivity to the media of ES-producing or non-producing cells was measured after 5-day cultivation using the Cell Titer 96R AQueous Non-radioactive cell proliferation assay (Promega, Madison, WI). The results $(n=4)$ were calculated as the percentage of HUVEC proliferation, taking the positive control (HUVEC cells cultured in M200) as $100 \%$.

Animal experiments. Six- to eight-week-old C57BL/6 female mice $(\mathrm{H}-2 \mathrm{~b})$ were purchased from Charles Rivers, Germany. All experiments were carried out in accordance with the Guidelines for Animal Experimentation valid in the Czech Republic. Cells for oncogenicity tests were trypsinized and washed 3 times with PBS. Two counts of MK16 cells $\left(5 \times 10^{5}\right.$ or $\left.5 \times 10^{6}\right)$ and two counts of TC- 1 cells $\left(5 \times 10^{4}\right.$ or $\left.5 \times 10^{5}\right)$, i.e. 10 and $100 \mathrm{TID}_{50}$, were used. The same cell counts were used for the inoculation of all of the four mouse clones used, i.e. $5 \times 10^{5}$ or $5 \times 10^{6}$ ME3 and ME9 cells and $5 \times 10^{4}$ or $5 \times 10^{5}$ TE2 and TE5 cells. The cells, in $150 \mu 1$ volumes of PBS, were injected subcutaneously (s.c.) into the right flank of the mice. 


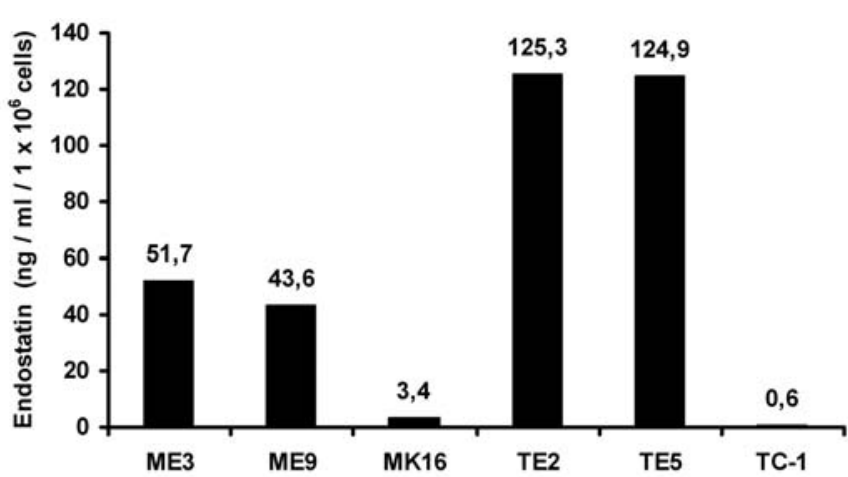

Figure 1. Production of ES by the parental MK16 and TC-1 cells and their ME3, ME9, TE2 and TE5 sublines as determined by ELISA.

Starting one week later, the mice were monitored for tumor appearance. To assess the effect of the ES-producing cells on metastasis formation by MK16-induced tumors, mice were inoculated s.c. with mixtures containing either $5 \times 10^{5}$ MK16

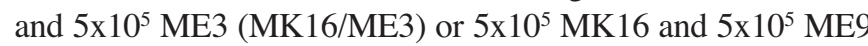
(MK16/ME9). Control groups of animals received only $5 \times 10^{5}$ MK16, $5 \times 10^{5}$ ME3 or $5 \times 10^{5}$ ME9 cells. Tumor diameters were measured with a calliper twice a week and when the tumor size reached $15 \mathrm{~mm}$ in its longest diameter the animals were humanely sacrificed, autopsied and inspected for the presence of metastasis on their lung surfaces. The tumor volume was expressed in $\mathrm{mm}^{3}$ using the height, width and length of tumor.

Screening of parental and ES-producing cells for angiogenic factors. The production of 25 angiogenic factors by MK16 and TC-1 cells and by the selected four ES-expressing sublines was evaluated using the Mouse Angiogenesis Antibody Array I (RayBiotech, Inc., Norcross, GA). Cultures at $80 \%$ confluency grown in $10-\mathrm{cm}$ dishes were processed according to the manufacturer's protocol. Lysates containing $500 \mu \mathrm{g}$ protein, as determined by the modified Bradford assay were used. The expression level of the angiogenic factors was determined by quantification of signals using Phoretix ${ }^{\mathrm{TM}} 2 \mathrm{D}$ Expression software. The values were normalized in accordance with the manufacturer's protocol, the positive control being taken as $100 \%$.

Western blot analysis. Cells $\left(3.5 \times 10^{5}\right)$ in D-MEM with $10 \%$ FCS were seeded onto culture dishes. After $48 \mathrm{~h}$ the cells were harvested, lysed in buffer containing 4\% SDS, $20 \%$ glycerol, $10 \%$ mercaptoethanol, $2 \mathrm{mM}$ EDTA and $100 \mathrm{mM}$ Tris $\mathrm{pH} 8.0$ and boiled for $5 \mathrm{~min}$. The lysates were subjected to $12 \%$ SDS-PAGE and transferred onto polyvinyl difluoride membranes (Amersham Hybond XL, UK). The membranes were incubated for $1 \mathrm{~h}$ with polyclonal rabbit anti-mouse IL- $1 \alpha$ antibody (Abcam, Cambridge, UK) diluted 1:2000 in 10\% skim milk. The membranes were then washed 5 times for 10 min in $10 \%$ Tween-20-PBS solution and blotted with horseradish-linked peroxidase donkey anti-rabbit IgG (Amersham Biosciences, Buckinghamshire, UK). The recombinant $17 \mathrm{kDa}$ mature form of IL-1 $\alpha$, provided by the manufacturer served as positive control. Development of the

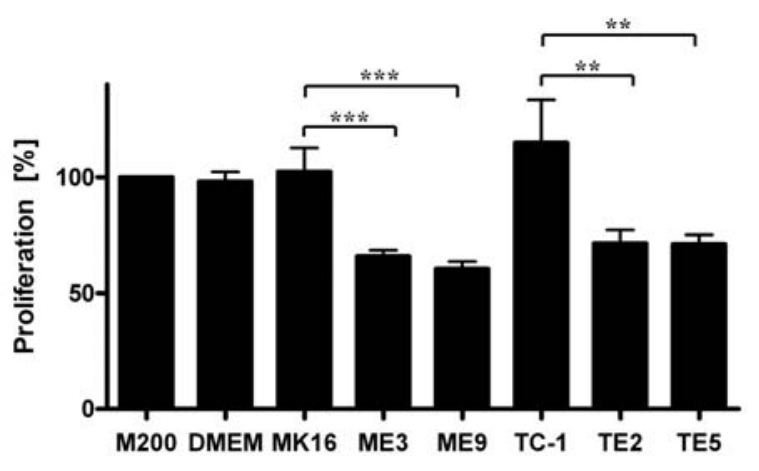

Figure 2. In vitro proliferation of HUVEC cells cultivated in media M200 and in media M200 supplemented with either concentrated D-MEM or concentrated media from the parental MK16 and TC-1 cells and their ESproducing ME3, ME9, TE2 and TE5 sublines. The bars indicate the means \pm SD. Statistical significance was determined by the Student unpaired t-test; ${ }^{* *} \mathrm{P}<0.001$ or ${ }^{* * *} \mathrm{P}<0.0001$. $(\mathrm{n}=4$, mean $\pm \mathrm{SD})$.

membranes was done with an ECL Western blotting detection system plus (Amersham Biosciences) according to the manufacturer's instructions.

Treatment of MK16 cells with conditioned media from ESproducing cells. MK16, ME3 and ME9 cells $\left(2 \times 10^{5}\right)$ were seeded in $25-\mathrm{cm}$ culture bottles. After $48 \mathrm{~h}$, the media from MK16 cultures were removed and replaced with conditioned media from either ME3 or ME9 cells. This procedure was repeated twice a day for 5 days. MK16 cells treated with media from MK16 cells kept in parallel served as a control. On the last day, after 3-h incubation with the fresh EScontaining or control media, the ES-treated and ES-untreated MK16 cells were harvested and tested by Western blotting as indicated above. ME3 and ME9 cells were tested in parallel.

Statistical methods. Statistical evaluation of tumor growth was made by two-way analysis of variance (ANOVA, logrank). Data obtained in the HUVEC proliferation assay and data on metastasis formation were analysed using the Student unpaired t-test. A difference between groups was considered significant at $\mathrm{p}<0.05$ on the Graphpad PRISM 4.0 program (Graphpad Software Inc., San Diego, CA).

\section{Results}

Generation of stable transfectants secreting mouse ES. After the plasmid pBLAST49-mEndo was transfected into MK16 or TC-1 cells, several blasticidin-resistant colonies formed. From each transfected line, two clones were selected at random for further experiments. Those derived from MK16 cells were denoted ME3 and ME9; those derived from TC-1 cells were designated TE2 and TE5. Their media were tested for the presence of ES by an ELISA assay. The results are shown in Fig. 1. The two MK16-derived clones produced $\sim 50 \mathrm{ng} / 10^{6}$ cells $/ 48 \mathrm{~h}$, while cell production by the two TC-1-derived clones was $\sim 2.5 \mathrm{x}$ higher. The production remained stable after five passages either in the presence or absence of blasticidin (results not shown). Growth curves of the original cells and their ES-producing clones were constructed. No significant differences were observed (results not shown). 
A)
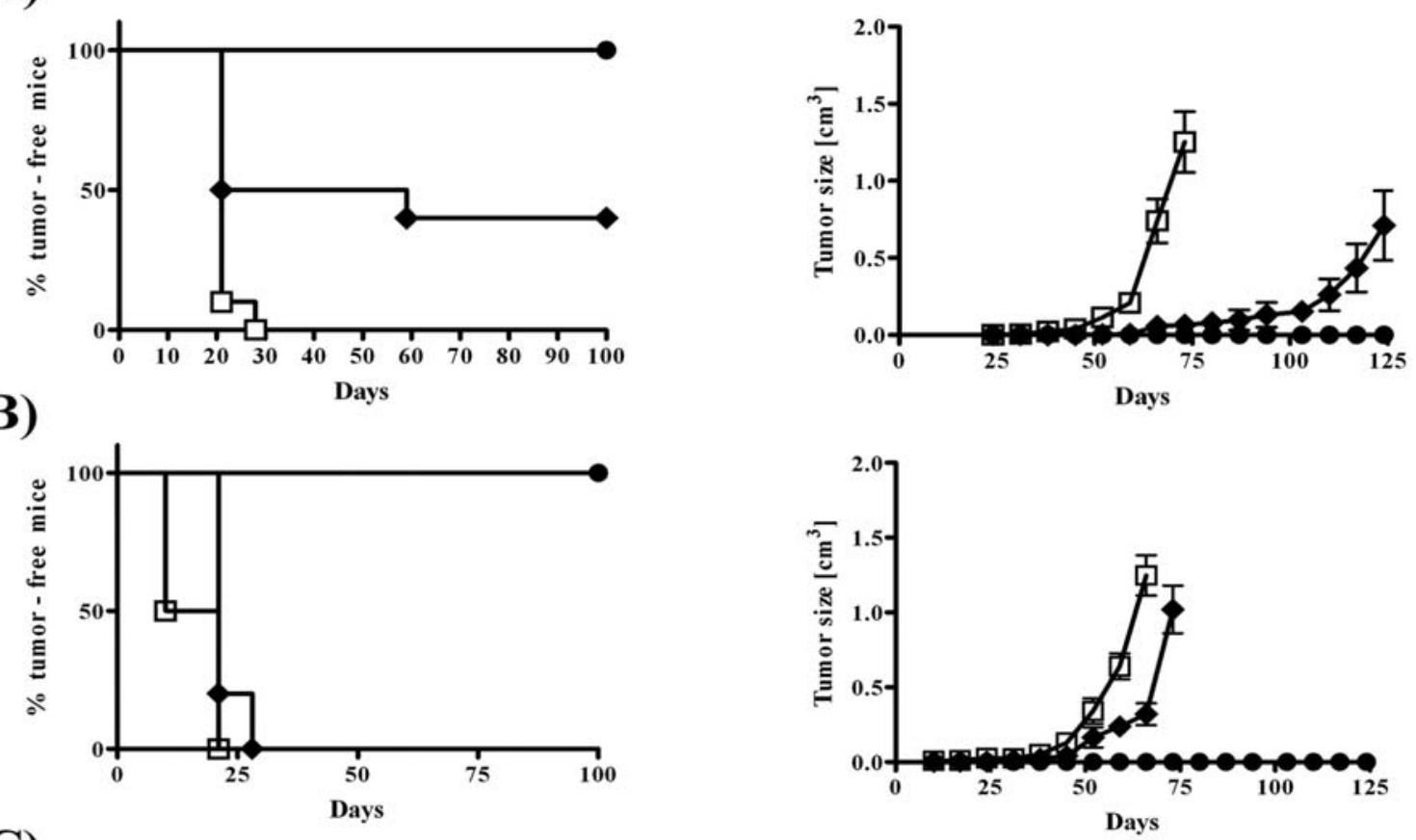

C)
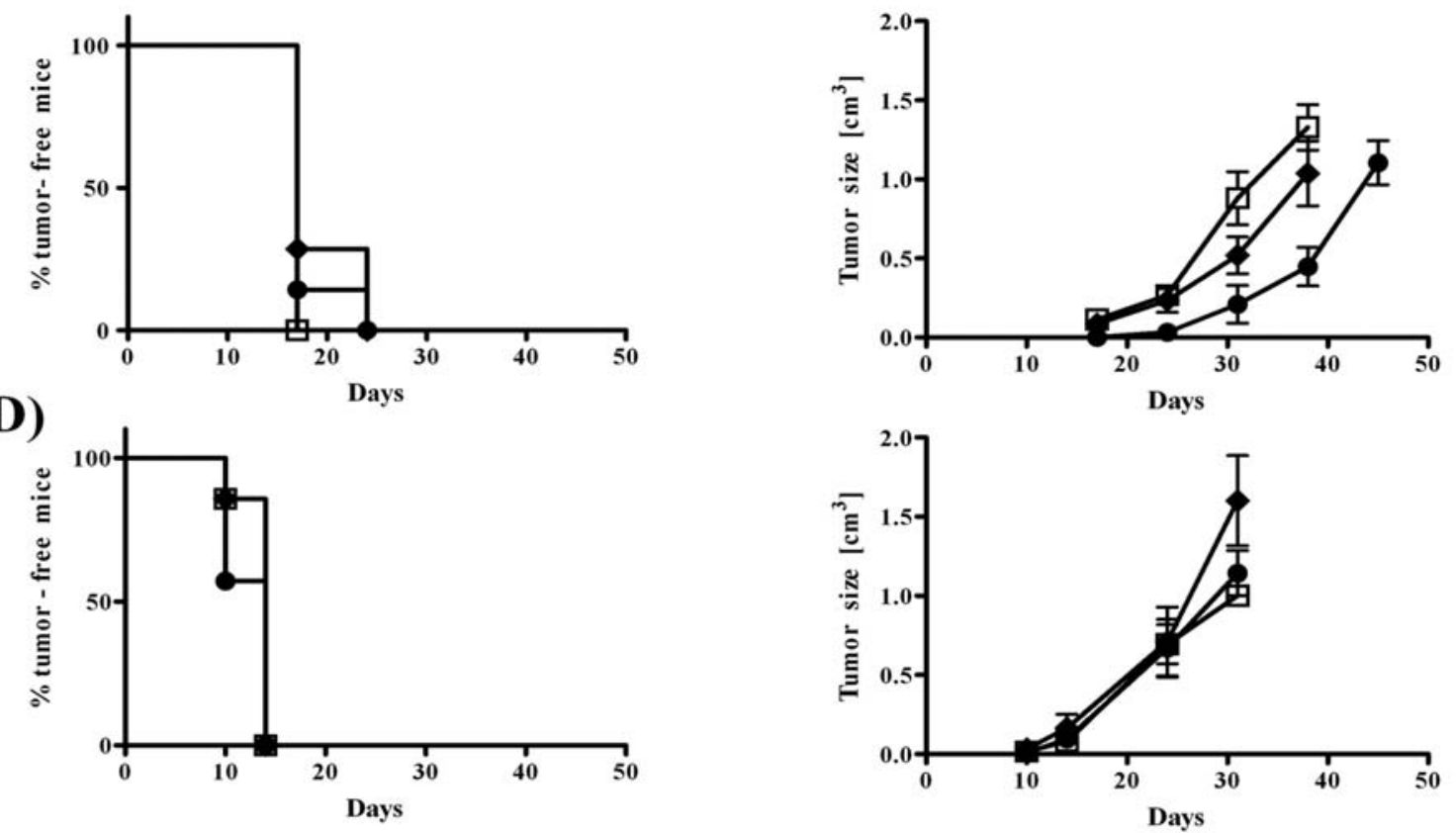

Figure 3. Tumor development in mice inoculated with MK16 $\left(5 \times 10^{5}\right.$ or $\left.5 \times 10^{6}\right)$ or TC-1 $\left(5 \times 10^{4}\right.$ or $\left.5 \times 10^{5}\right)$ cells. The same cell counts were used for the inoculation of the respective cell clones, i.e. counts of $5 \times 10^{5}$ or $5 \times 10^{6}$ for ME3 and ME9 cells and $5 \times 10^{4}$ or $5 \times 10^{5}$ for TE2 and TE5 cells. (A) Tumor development (left) and growth (right) in mice inoculated with MK16 cells and their ES-producing sublines. (B) Tumor development (left) and growth (right) in mice inoculated with TC-1 cells and their ES-producing sublines. The bars indicate the means \pm SD. (A) $\square-$ MK16 5x105, (0/10); $\bullet-$ ME3 5x105, (10/10);

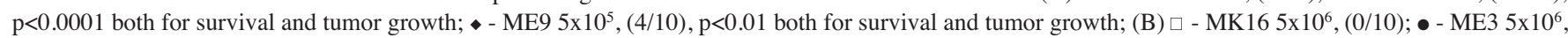

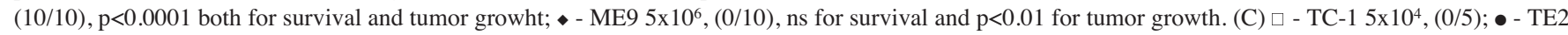
$5 \times 10^{4},(0 / 5)$, ns for survival, $\mathrm{p}<0.01$ for tumor growth; - TE5 $5 \times 10^{4},(0 / 5)$, ns for survival, $\mathrm{p}<0.05$ for tumor growth; (D) $\square-\mathrm{TC}-15 \times 10^{5},(0 / 5)$; $\bullet-$ TE2 $5 \times 10^{5},(0 / 5)$, ns both for survival and tumor growth ; - TE5 $5 \times 10^{5},(0 / 5)$, ns both for survival and tumor growth.

Expression of MHC class I molecules by ES-producing cells. As already mentioned, MK16 cells have a downregulated in vitro expression of MHC class I molecules. We tested the expression of MHC class I in all of the transduced cells, using the MK16 cells as a negative control and TC-1 cells as a positive control. The genetic modification, i.e. ES production and blasticidin resistance, was not associated with any changes in the expression of MHC class I molecules in either the MK16 or TC-1 derivatives (results not shown).

Inhibition of HUVEC cell proliferation by products of ESproducing cells. To prove that the ES produced by the genemodified cells was biologically active, we tested the capability of the concentrated media to suppress the proliferation of 
A)
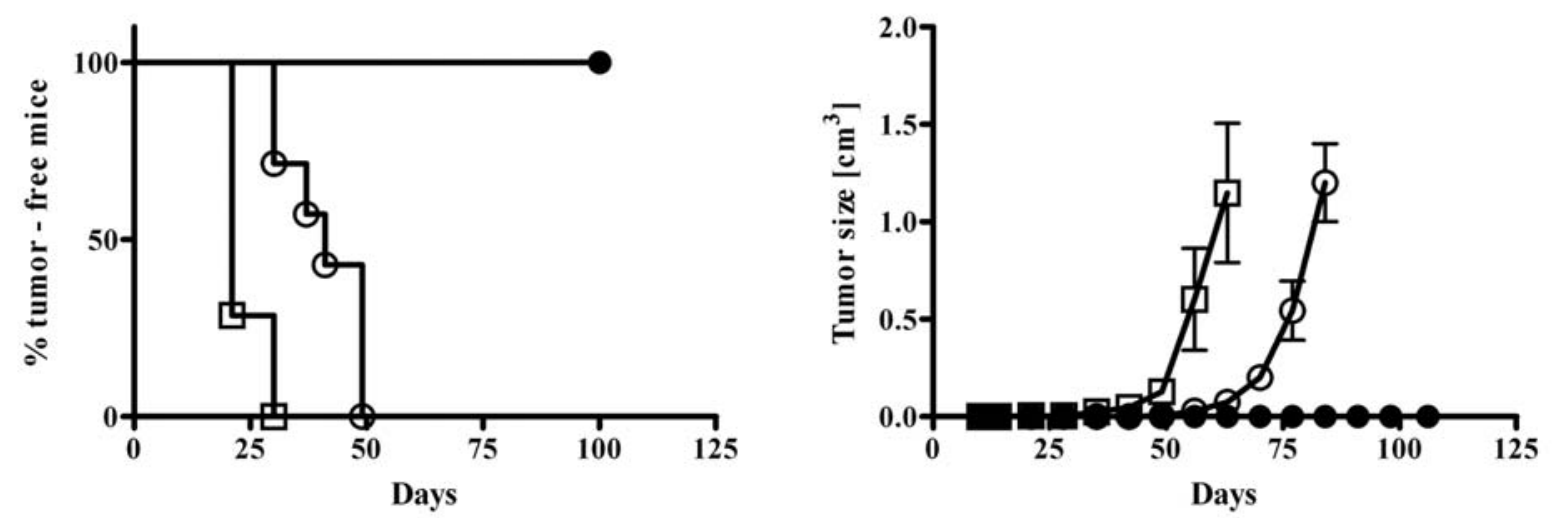

B)
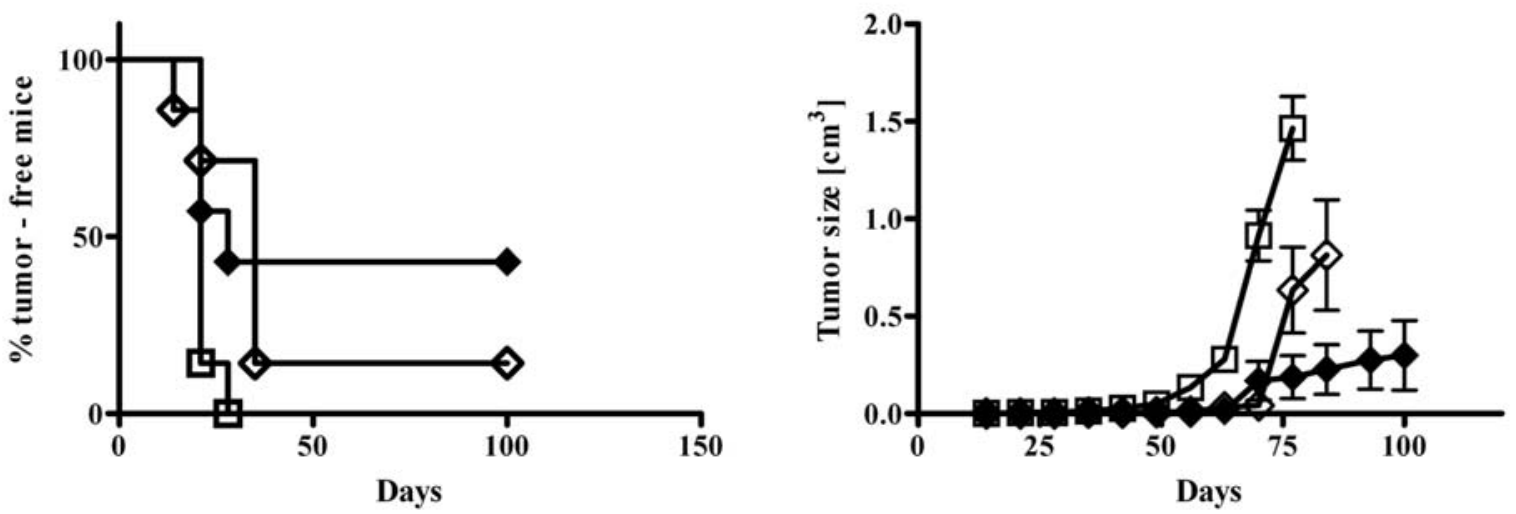

Figure 4. Tumor development in mice inoculated with $5 \times 10^{5}$ MK16 cells, their ES-producing ME3 or ME9 sublines, and with mixtures of 5x105 MK16 cells

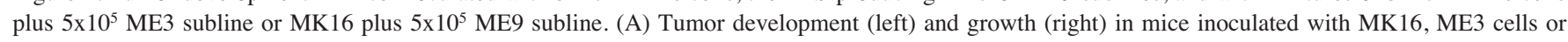
their mixture. (B) Tumor development (left) and growth (right) in mice inoculated with MK16 cells, ME9 cells or their mixture. The bars indicate the means \pm

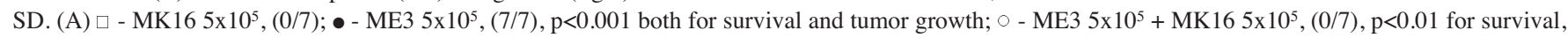

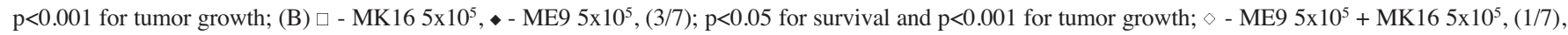
$\mathrm{p}<0.05$ for both survival and tumor growth.

HUVEC cells. Similarly conditioned media from the parental cells served as controls. The results are shown in Fig. 2. M200 mixed with concentrated D-MEM alone did not have any influence on HUVEC cell proliferation as compared with cells cultured in M200. No anti-proliferative effect was induced by processed media taken from either MK16 or TC-1 cell cultures. However, the proliferative activity of HUVEC cells was significantly reduced when cultivated in the presence of media taken from the ES-producing cells. The test was repeated twice and the results were similar.

Oncogenicity and metastizing activity of ES-producing cells. Tumor development after injection of two different doses of each of the four ES-producing cell lines and the parental cells is shown in Fig. 3. ME3 cells exhibited no oncogenic activity in either cell dose used $\left(5 \times 10^{5}\right.$ or $\left.5 \times 10^{6}\right)$. The lower dose $\left(5 \times 10^{5}\right)$ of ME9 cells resulted in tumor development in 6 out of 10 mice, while all animals (10/10) developed tumors after inoculation with the higher dose $\left(5 \times 10^{6}\right)$, though at a slower rate than animals inoculated with a corresponding dose of the parental MK16 cells. This difference was significant, more so in the case of the lower cell dose (Fig. 3A). Furthermore, the number of lung metastases were reduced in animals bearing ME9-induced tumors. On the other hand, tumors developed in all animals inoculated with either TE2 or TE5 cells, although the tumor formation was delayed when the lower cell dose was used (Fig. 3B). From tumors formed by ME9, TE2 and TE5 cells, cell lines were isolated and tested for ES production. They were all positive (results not shown), this indicating that the tumor formation was not due to the loss of ES production.

Effect of simultaneous administration of MK16 cells and either ME3 or ME9 cells on metastasis formation. As mentioned above, tumors induced by MK16 cells spontaneously metastasize to the lungs. We examined whether the ES-producing ME9 cells (which had retained the oncogenic phenotype) or ME3 cells (which had lost the oncogenic phenotype) had any impact on metastasis formation after having been inoculated in a mixture with MK16. Mice were inoculated with mixtures containing either $5 \times 10^{5}$ MK16 and $5 \times 10^{5}$ ME3 cells (MK16/ ME3) or $5 \times 10^{5}$ MK16 and $5 \times 10^{5}$ ME9 cells (MK16/ME9). Control groups of animals were inoculated with only MK16, ME3 or ME9 cells. The results are shown in Fig. 4. Tumors 


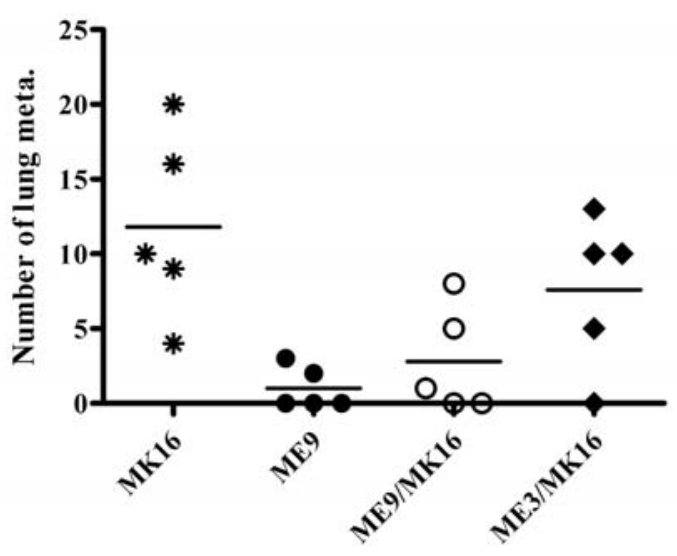

Figure 5. Formation of metastases in mice inoculated with $5 \times 10^{5}$ MK16 cells, their ES-producing ME3 or ME9 subline or a mixture of MK16 cells with either ME3 or ME9 cells. The animals were humanely sacrificed when the size of the tumor reached $15 \mathrm{~mm}$ in its longest diameter, autopsied and their lung metastases were counted. * MK16 cells, $\bullet$ - ME9 cells, $\mathrm{p}<0.01, \circ-$ MK16 + ME9 cells, p $<0.05$, - MK16 + ME9 cells, ns. ME3 cells administered in parallel did not induce tumours (not shown).

were detected in 4 out of 7 animals inoculated with ME9 cells, whereas no ME3-inoculated animals developed tumors. All animals inoculated with MK16 cells or a mixture of M16 and ME3 cells developed tumors; however, tumor development was markedly delayed in the latter group. Tumor development was also delayed in mice which had received a mixture of MK16 and ME9 cells, with one animal in this group remaining tumor-free. The tumor-bearing animals were sacrificed and autopsied when their tumor size had reached $15 \mathrm{~mm}$ in its longest diameter. The groups differed in the occurrence and number of lung metastases. As shown in Fig. 5, all mice inoculated with MK16 alone and all but one inoculated with the MK16/ME3 mixture developed multiple metastases. From one of these metastases a cell line was isolated and tested for the production of ES. The result was negative, this indicating that this and most likely also the other metastases in these animals were caused by the parental MK16 cells. In agreement with previous results, the majority of mice inoculated with ME9 cells remained free of metastases, while the positive animals had only a few metastases each. The number of metastases was significantly reduced also in animals inoculated with the ME9/MK16 mixture when compared with animals inoculated with MK16 cells.

Production of angiogenic factors by parental cells and ESproducing cells. In an attempt to correlate the differences in pathogenicity detected in vivo with the in vitro characteristics of the cells under investigation, we undertook to determine, in the cell lysates, the content of 25 cell factors known to be involved in angiogenesis by using the Mouse Angiogenesis Antibody Array (MAAA) kit I. Lysates from all six cell lines were tested simultaneously, using membranes originating from the same production batch. The amounts of all the 25 angiogenesis factors tested in the parental TC-1 and MK16 cells are indicated in Fig. 6A. It is apparent that higher levels of most of the angiogenic factors were detected in the MK16 than in the TC- 1 cells. The most pronounced difference was detected in the content of IL- $1 \alpha$, its content being markedly higher in the MK16 than the TC-1 cells. When MK16 cells were compared with their ES-producing sublines, the most significant difference was a reduction in the IL- $1 \alpha$ content in the sublines. Also reduced, though to a lesser extent, were the contents of some other angiogenesis-associated factors, viz. the basic fibroblast growth factor (bFGF), granulocyte colony stimulation factor (G-CSF), interleukin-12 (IL-12), interleukin-13 (IL-13), monocyte chemoattractact protein-1 (MCP-1), macrophage colony stimulating factor (M-CSF), platelet factor 4 (PF4), tissue inhibitor of metalloproteinases-2 (TIMP-2) or thrombopoietin (TRP) (Fig. 6B). There was a much smaller difference between the parental TC-1 cells and their ES-producing sublines TE2 and TE5 (Fig. 6C). The test was repeated once more using cells of different passage levels and a set of membranes from a different production batch. The results from the two experiments were somewhat different, e.g. higher contents of tissue inhibitors of metalloproteinases were detected in the repeated test in all of the six lines tested, but the differences in the IL- $1 \alpha$ content as detected in the first experiment were observed again. To verify the findings obtained with the MAAA kit, we undertook to detect the intracellular IL-1 $\alpha 33 \mathrm{kDa}$ precursor by Western blotting in lysates of all six cell lines using polyclonal anti-IL- $1 \alpha$ antibody. The $17 \mathrm{kDa}$ mature recombinant IL- $1 \alpha$ provided by the manufacturer served as a positive control. The results are shown in Fig. 7. It is evident that the MK16 cells, but neither ME3 nor ME9 subline, contained demonstrable amounts of the IL- $1 \alpha 33 \mathrm{kDa}$ precursor. IL-1 $\alpha 33 \mathrm{kDa}$ precursor was not detected in lysates of TC-1 and their ES-producing sublines tested in parallel. Similar results were obtained in repeated tests.

To gain more information on the mechanism of suppression of IL-1 $\alpha$ production in the ES-producing cells, we exposed the parental MK16 cells to conditioned media from the ES-producing ME3 and ME9 cell cultures as described in Materials and methods. The results of Western blotting are presented in Fig. 8. It can be seen, that the production of the cytokine was strongly suppressed by the treatment with the ES-containing media.

\section{Discussion}

Gene-transfer strategies (GTS) have been extensively used in the study of the effect of a variety of anti-angiogenic factors, primarily endostatin (ES), on tumor growth (11-14). It is believed that this approach might enable large production of ES, which is a precondition for its extensive use in cancer treatment. Where GTS are used, there is no danger of denaturation, such as is involved in the preparation of recombinant ES, and, since successfully transfected cells maintain ES production over long periods of time, there should not be a need for frequent administration of the drug. Gene-modified tumor cells constitutively expressing ES have been studied, but rarely $(15,16)$. The oncogenicity of such cells has been reduced and progression of micrometastases to macroscopic disease has been prevented; however, to the best of our knowledge, their biology has not yet been investigated in much detail. In the present study, we report on the behaviour of two mouse oncogenic, HPV16-transformed cell lines, viz. MK16 and TC-1, permanently transduced to produce ES. Our data indicate that the biological changes associated with 


\section{A)}

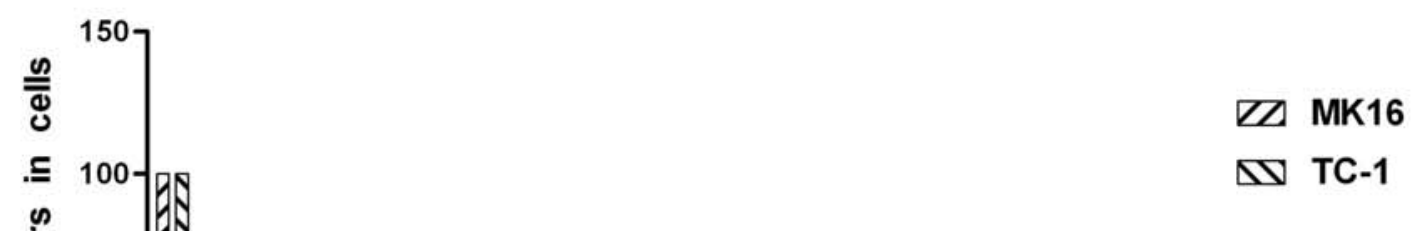

B)

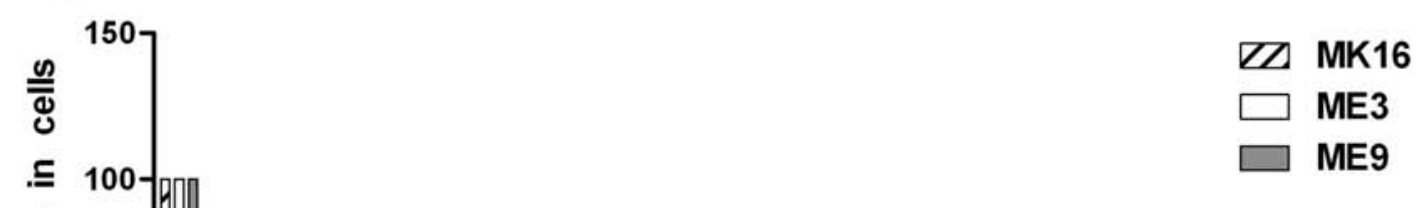

C)

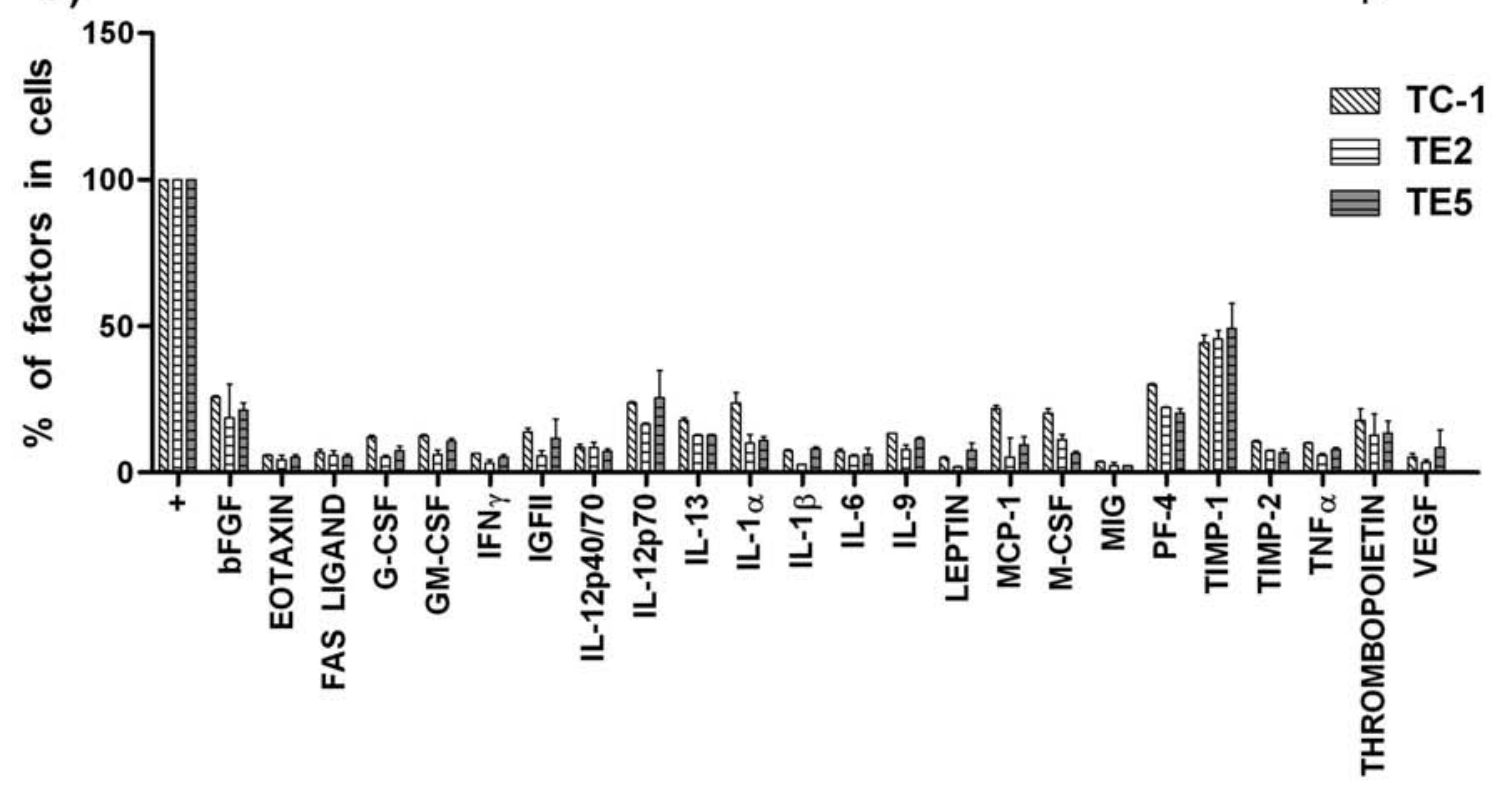

Figure 6. Production of 25 factors known to be involved in angiogenesis by MK16 and TC-1 cells and their ES-producing ME3, ME9, TE2 and TE5 sublines, as determined in cell lysates by the Mouse Angiogenesis Antibody Array I. (A) Comparison of TC-1 and MK16 cells. (B) Comparison of MK16, ME3 and ME9 cells. (C) Comparison of TC-1, TE2 and TE5 cells. 


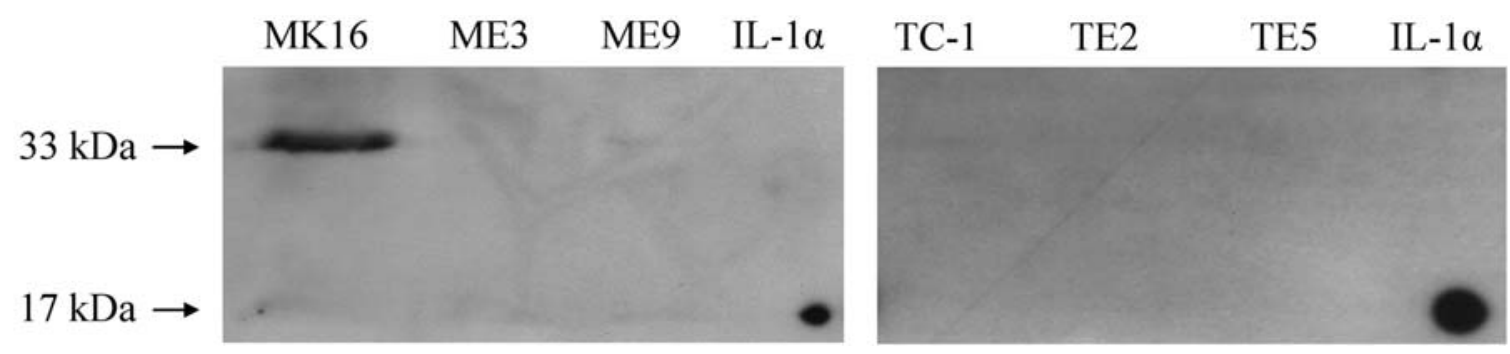

Figure 7. IL-1 $\alpha$ production by MK16 and TC-1 cells and their ES-producing ME3, ME9, TE2 and TE5 sublines as determined by Western blotting. The 33 kDa precursor was detected by rabbit anti-IL-1 $\alpha$ antibody (diluted 1:2000), $17 \mathrm{kDa}$ recombinant mature IL-1 $\alpha$ served as positive control.

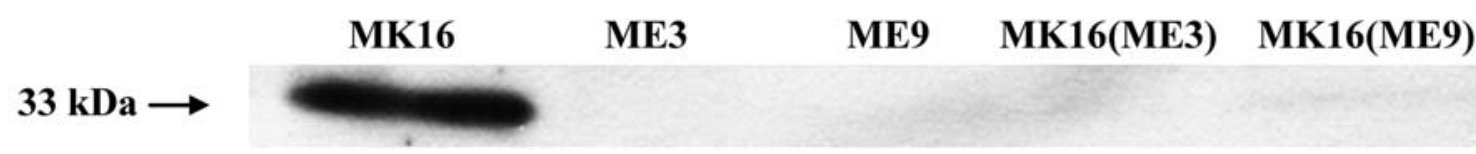

Figure 8. IL-1 $\alpha$ production by MK16, ME3 and ME9 cells and MK16 cells treated for five days with conditioned media from ES-producing ME3 (MK16ME3) and ME9 (MK16ME9) cells.

ES expression markedly differed between the progenies of these two cell lines and suggest that these differences were associated with the properties of the parental cells.

From the biological point of view, the most distinctive was the difference in oncogenicity and metastizing activity. ES production was associated with complete loss of oncogenic activity and metastatic potential in one of the MK16-derived sublines, designated ME3, and in another one, denoted ME9, with a significant reduction of oncogenicity and marked decrease of metastatic potential. It may be of interest that in some of the repeated tests a higher production of ES was detected in the ME3 than in ME9 cells (results not shown). If so, this might well explain the observed difference in their in vivo properties. On the other hand, the oncogenicity of both of the ES-producing TC-1-derived sublines tested remained almost intact, although these cells produced more ES than the corresponding MK16-derived cells. It has been shown that the dose-response curve of tumors to ES is U-shaped (17), this implying that high concentrations of ES might be less effective than lower ones. On the contrary, the difference encountered might be also associated, to a certain degree, with the different growth rate of the TC-1 and MK-16-induced tumors in vivo. For fast-growing tumors, higher doses of ES are required (18). As shown in the Results section above, the tumor size required for euthanasia $(15 \mathrm{~mm}$ in the longest diameter of the tumor), as used in the present experiments, was reached in 50-60 days in the case of MK16 cells and in 2030 days in the case of TC- 1 cells. The difference may also be associated with the presence of ES receptors in MK16 cells (see below) and their absence or decreased amount in TC-1 cells, however, no direct evidence on this is available at present.

It has also been reported that ES failed to inhibit tumor growth owing to an unusually high upregulation of proangiogenic factors in the respective tumor cells (19). Although we did not obtain any evidence that the resistance of TC- 1 cells to ES effects was due to such a condition (see Fig. 6, and see below), we cannot rule out the involvement of other angiogenesis-associated factors, not tested in the present study. Of special interest may be the outcome of the present experiments in which the oncogenicity and metastatic potential of mixtures of the ES-producing cells and the parental MK16 cells were tested. Although the admixture of ME9 to the parental MK16 cells only delayed subcutaneous tumor formation, it strongly reduced the development of lung metastases. The simultaneous inoculation of the non-oncogenic ME3 and the MK16 cells produced a much lesser effect in these animals: metastases developed more frequently than in MK16/ME9 inoculated animals. It is likely that these developments were associated with the lability of mouse ES, which has a circulatory half-life of 0.6-3 h (20). A continuous delivery of ES can supposedly be better ensured by the sustained, though slow, growth of ME9 cells in vivo (finally resulting in tumor development) than by the possibly rapidly rejected ME3 cells. Preliminary results demonstrating resistance of ME3-treated animals to the challenge with the parental MK16 cells suggest that this may be the case (unpublished data). A possible role of ES in immunological reactions has previously been suggested (21).

To further analyse the possible reasons for the different behaviour of the cells examined, we tested their lysates for the contents of 25 factors known to be involved in angiogenesis. We were aware that the interpretation of any findings would be difficult for at least two reasons. First, many of the factors tested are secretory and their contents in the cell lysates would not reflect their real production. Second, many more factors than those we were able to test are known to be involved in angiogenesis. The most conspicuous difference between MK16 cells and their $\mathrm{ES}^{+}$progenies was a markedly reduced production of IL- $1 \alpha$ by the latter, as was shown with the use of both the MAAA kit and Western blotting. A similar difference was found between the two parental cell lines, MK16 (high production) and TC-1 (low or no production), but not between the parental TC-1 cells and their ES ${ }^{+}$ derivatives. IL- $1 \alpha$ and IL-1ß belong among the most potent, multifunctional cell activators (22). In addition to influencing 
inflammatory processes, they induce a variety of growthpromoting, but also some degradative, processes. IL-1ß, which is secreted in response to inflammatory signals, is active only extracellularly. IL-1 $\alpha$ mainly remains cell-associated, being expressed in its cleaved $17 \mathrm{kDa}$ form on the cell surface, but it also acts, in its precursor $33 \mathrm{kDa}$ form, as an intracrine messenger controlling genes involved in growth stimulation and cell differentiation. Thus, its content in the cell lysates reliably reflects its production rate. IL- $1 \alpha$ is only rarely secreted by normal cells, but is frequently secreted by tumor cells.

It has been reported previously that IL- $1 \alpha$ and IL- $1 \beta$ knock-out mice exhibited impaired blood vessel growth and tumor development (23). Although in some tumor systems the pro-angiogenic activity of IL- $1 \alpha$ was apparently less important than that of IL-1ß, in some other the reverse appeared to be true. Secreted IL- $1 \alpha$ has been shown to participate in the upregulation of the production of cytokines and in activation of pro-metastatic and anti-apoptotic genes in various tumor systems $(24,25)$, and a correlation between the presence of this cytokine and metastasis formation has been demonstrated in human gastric cancer (26). It has also been reported that cells transduced with IL-1 $\alpha$ gene were more invasive than the parental tumor cells $(27,28)$. Most recently, the investigation of cell lines derived from either metastizing or non-metastizing gastric and pancreatic cancers has revealed a strong association between increased IL- $1 \alpha$ expression levels and metastatic potential $(29,30)$. One may therefore concede that some of the phenomena observed in the present series of experiments might be well explained by the rate of IL-1 $\alpha$ production. If our reasoning is correct, then the effects of ES on tumor growth need not be limited to endothelial cells, as it was suspected for a long time, but might be mediated by changes directly induced in the tumor cells by an autocrine or intracrine mechanism. The results we obtained with parental MK16 cells treated with ES-containing media of the ME3 and ME9 cells indicate that an autocrine mechanism was involved and seem to indicate that ES receptors, which have not been clearly defined as yet, were present at the MK16 cell surface. This possibility is supported by the recent observations of direct inhibitory effects of ES on the behaviour of other cancer cells in vivo $(31,32)$, although it has had no effect on the replication of tumor cells in vitro (33) (also our own data, unpublished). In the present experiments, in addition to IL- $1 \alpha$, a decreased production of some other angiogenic factors was also detected in MK-16-derived $\mathrm{ES}^{+}$cell lines. The reductions of these other factors were lesser than in the case of IL-1 $\alpha$, even being on the brink of significance in some instances. However, their cumulative deficiency could also contribute to the reduction of oncogenicity and metastatic activity.

To summarize, in the present study we showed that ES gene transduction was associated with the loss or marked decrease of oncogenicity including the metastatic potential in the derivatives of one, viz. the MK16 cells, but not the other, viz. the TC- 1 cells. These two cell lines differed in a number of properties including a different expression of several factors involved in angiogenesis of which the most marked was an increased expression of IL- $1 \alpha$ by MK16 cells. The decrease of oncogenicity of their ES-producing sublines was associated with a marked suppression of IL-1 $1 \alpha$ production. These data suggest that the increased production of IL-1 $1 \alpha$ (and possibly some other pro-angiogenic factors) by MK16 cells was associated with the metastatic potential of the tumors induced by these cells, and that the ES production resulting in IL- $1 \alpha$ suppression was directly involved in the the marked attenuation of MK16-derived progenies. The present results seem to provide leads for further studies aimed at clarifying the mechanisms of ES action and its possible use in the treatment of cancer. Experiments to analyse the connection between IL-1 $\alpha$ production and the susceptibility of the cell oncogenicity to ES and to explain the phenomena involved in a reasonable way are under preparation and, partially, already under way.

\section{Acknowledgements}

This study was supported by the Grant No. NR/9075-3 of the Internal Granting Agency of the Ministry of Health of the Czech Republic.

\section{References}

1. O'Reilly MS, Boehm T, Shing Y, Fukai N, Vasios G, Lane WS, Flynn E, Birkhead JR, Olsen BR and Folkman J: Endostatin: an endogenous inhibitor of angiogenesis and tumor growth. Cell 88: 277-285, 1997.

2. Abdollahi A, Hahnfeldt P, Maercker C, Gröne HJ, Debus J, Ansorge W, Folkman J, Hlatky L and Huber PE: Endostatin's antiangiogenic signaling network. Mol Cell 13: 649-663, 2004.

3. Kim YM, Jang JW, Lee OH, Yeon J, Choi EY, Kim KW, Lee ST and Kwon YG: Endostatin inhibits endothelial and tumor cellular invasion by blocking the activation and catalytic activity of matrix metalloproteinase. Cancer Res 60: 5410-5413, 2000

4. Schuch G, Heymach JV, Nomi M, Machluf M, Force J, Atala A, Eder JP, Folkman J and Soker S: Endostatin inhibits the vascular endothelial growth factor-induced mobilization of endothelial progenitor cells. Cancer Res 63: 8345-8350, 2003.

5. Dixelius J, Cross M, Matsumoto T, Sasaki T, Timpl R and Claesson-Welsh M: Endostatin regulates endothelial cell adhesion and cytoskeletal organization. Cancer Res 62: 1944-1947, 2002.

6. Dhanabal M, Ramchandran R, Waterman MJ, Lu H, Knebelmann B, Segal M and Sukhatme VP: Endostatin induces endothelial cell apoptosis. J Biol Chem 274: 11721-11726, 1999.

7. Smahel M, Sobotkova E, Bubenik J, Simova J, Zak R, Ludvikova V, Hajkova R, Kovarik J, Jelinek F, Povysil C, Marinov J and Vonka V: Metastatic MHC class I-negative mouse cells derived by transformation with human papillomavirus type 16. Br J Cancer 84: 374-380, 2001.

8. Lin KY, Guarnieri FG, Staveley-O'Carroll KF, Levitsky HI, August JT, Pardoll DM and Wu TC: Treatment of established tumors with a novel vaccine that enhances major histocompatibility class II presentation of tumor antigen. Cancer Res 56: 21-26, 1996.

9. Janouskova O, Sima P and Kunke D: Combined suicide gene and immunostimulatory gene therapy using AAV-mediated gene transfer to HPV16 transformed mouse cell: decrease of oncogenicity and induction of protection. Int J Oncol 22: 569-577, 2003.

10. Mikyskova R, Bubenik J, Vonka V, Smahel M, Indrova M, Bieblova J, Simova J and Jandlova T: Immune escape phenotype of HPV16-associated tumours: MHC class I expression changes during progression and therapy. Int J Oncol 26: 521-527, 2005 .

11. Chen QR, Kumar D, Stass SA and Mixson AJ: Liposomes complexed to plasmids encoding angiostatin and endostatin inhibit breast cancer in nude mice. Cancer Res 59: 3308-3312, 1999.

12. Feldman AL, Restifo NP, Alexander HR, Bartlett DL, Hwu P, Seth P and Libutti SK: Antiangiogenic gene therapy of cancer utilizing a recombinant adenovirus to elevate systemic endostatin levels in mice. Cancer Res 60: 1503-1506, 2000.

13. Sauter BV, Martinet O, Zhang WJ, Mandeli J and Woo SL: Adenovirus-mediated gene transfer of endostatin in vivo results in high level of transgene expression and inhibition of tumor growth and metastases. Proc Natl Acad Sci USA 97: 4802-4807, 2000. 
14. Bazan-Peregrino M, Seymour LW and Harris AL: Gene therapy targeting to tumor endothelium. Cancer Gene Ther 14: 117-127, 2007.

15. Yoon SS, Eto H, Lin CM, Nakamura H, Pawlik TM, Song SU and Tanabe KK: Mouse endostatin inhibits the formation of lung and liver metastases. Cancer Res 59: 6251-6256, 1999.

16. Peroulis I, Jonas $\mathrm{N}$ and Saleh M: Antiangiogenic activity of endostatin inhibits C6 glioma growth. Int J Cancer 97: 839-845, 2002.

17. Celik I, Sürücü O, Dietz C, Heymach JV, Force J, Höschele I, Becker CM, Folkman J and Kisker O: Therapeutic efficacy of endostatin exhibits a biphasic dose-response curve. Cancer Res 65: 11044-11050, 2005.

18. Prox D, Becker C, Pirie-Shepherd SR, Celik I, Folkman J and Kisker O: Treatment of human pancreatic cancer in mice with angiogenic inhibitors. World J Surg 27: 405-411, 2003.

19. Cui R, Takahashi K, Takahashi F, Tanabe KK and Fukuchi Y: Endostatin gene transfer in murine lung carcinoma cells induces vascular endothelial growth factor secretion resulting in upregulation of in vivo tumorigenecity. Cancer Lett 232: 262-271, 2006.

20. Cho HM, Rosenblatt JD, Kang YS, Iruela-Arispe ML, Morrison SL, Penichet ML, Kwon YG, Kim TW, Webster KA, Nechustan H and Shin SU: Enhanced inhibition of murine tumor and human breast tumor xenografts using targeted delivery of an antibody-endostatin fusion protein. Mol Cancer Ther 4: 956-967, 2005.

21. Kawashima H, Waranabe N, Hirose M, Sun X, Atarashi K, Kimura T, Shikata K, Matsuda M, Oqava D, Hejasvaara R, Rehn M, Philajaniemi T and Miyasaka M: Collagen XVIII, a basement membrane heparan sulfate proteoglycan, interacts with L-selectin and monocyte chemoattractant protein-1. J Biol Chem 278: 13069-13076, 2003.

22. Apte RN, Dotan S, Elkabets M, White RW, Reich E, Carmi Y, Song X, Dvozkin T, Krelin Y and Voronov E: The involvement of IL-1 in tumorigenesis, tumor invasiveness, metastasis and tumor-host interactions. Cancer Metastasis Rev 25: 387-408, 2006.

23. Voronov E, Shouval DS, Krelin Y, Cagnano E, Benharroch D, Iwakura Y, Dinarello CA and Apte RN: IL-1 is required for tumor invasiveness and angiogenesis. Proc Natl Acad Sci USA 100: $2645-2650,2003$
24. Lazar-Molnar E, Hegyesi H, Toth S and Falus A: Autocrine and paracrine regulation by cytokines and growth factors in melanoma. Cytokine 12: 547-554, 2000.

25. Nozaki S, Sledge GW and Nakshatri H: Cancer cell-derived interleukin 1 alpha contributes to autocrine and paracrine induction of pro-metastatic genes in breast cancer. Biochem Biophys Res Commun 275: 60-62, 2000.

26. Tomimatsu S, Ichikura $\mathrm{T}$ and Mochizuki H: Significant correlation between expression of of interleukin 1 alpha and liver metastasis in gastric carcinoma. Semin Cancer Biol 112: 277-290, 2002.

27. Apte RN and Voronov E: Interleukin 1: a major pleiotropic cytokine in tumor-host interactions. Semin Cancer Biol 12: 277-290, 2002.

28. Chirivi RG, Chiodoni C, Musiani P, Garofalo A, Bernasconi S, Colombo MP and Ciavazzi R: IL-alpha-gene transfected human melanoma cells increase tumor-cell adhesion to endothelial cells and their retention in the lung of nude mice. Int J Cancer 67: 856-863, 1996.

29. Ma J, Sawai H, Matsuo Y, Ochi N, Yasuda A, Takahashi H, Wakasugi T, Funahashi H, Sato M, Okada Y, Takeyama H and Manabe T: Interleukin- $1 \alpha$ enhances angiogenesis and is associated with liver metastatic potential in human gastric cancer cell lines. J Surg Res 148: 197-204, 2008

30. Matsuo Y, Sawwai H, Ochi N, Yasuda A, Takahashi H, Funahashi $\mathrm{H}$, Takeyama $\mathrm{H}$ and Guha S: Interleukin $-1 \alpha$ secreted by pancreatic cancer cells promotes angiogenesis and its therapeutic implications. J Surg Res 153: 274-281, 2009.

31. Wilson RF, Morse MSA, Pei P, Renner RJ, Schuller DE, Robertson FM and Mallery SM: Endostatin inhibits migration and invasion of head and neck squamous cell carcinoma cells. Anticancer Res 23: 1289-1295, 2003.

32. Yokoyama Y, Sedgewick G and Ramakrishnan S: Endostatin binding to ovarian cancer cells inhibits peritoneal attachement and dissemination. Cancer Res 67: 10813-10822, 2007.

33. Folkman J: Antiangiogenesis in cancer therapy - endostatin and its mechanisms of action. Exp Cell Res 312: 594-607, 2006. 